\title{
MANEJO DA ASMA AGUDA EM ADULTOS NA SALA DE EMERGÊNCIA: EVIDÊNCIAS ATUAIS
}

\author{
Paulo de Tarso Roth Dalcin*1, Christiano Perin ${ }^{2}$
}

Trabalho realizado no Serviço de Pneumologia do Hospital de Clínicas de Porto Alegre (HCPA) - Faculdade de Medicina, Universidade Federal do Rio Grande do Sul (UFRGS), Porto Alegre, RS

\author{
*Correspondência: \\ Rua Honório Silveira Dias, \\ 1529/901 - Bairro São João \\ CEP 90540-070 - Porto \\ Alegre - RS \\ Tel/Fax: (51) 3330-0521 \\ pdalcin@terra.com.br
}

\begin{abstract}
RESUMO
Asma é uma doença com uma alta prevalência em nosso meio e ao redor do mundo. Embora novas opções terapêuticas tenham sido recentemente desenvolvidas, parece haver um aumento mundial na sua morbidade e mortalidade. Em muitas instituiçōes, as exacerbações asmáticas ainda se constituem em uma emergência médica muito comum. As evidências têm demonstrado que o manejo da asma aguda na sala de emergência concentra decisões cruciais que podem determinar o desfecho desta situação clínica. Nesta revisão, enfocaremos a avaliação e o tratamento do paciente com asma aguda na sala de emergência, descrevendo uma estratégia apropriada para o seu manejo. Serão consideradas as seguintes etapas: diagnóstico, avaliação da gravidade, tratamento, avaliação das complicações, decisão sobre onde se realizará o tratamento adicional e orientações por ocasião da alta da emergência. Espera-se que estas recomendações contribuam para que o médico clínico tome as decisões apropriadas durante o manejo da asma aguda na sala de emergência.
\end{abstract}

Unitermos: Asma. Medicina de emergência. Tratamento de emergência. Procedimentos clínicos.

\section{INTRODUÇÃO}

Asma é uma doença inflamatória crônica das vias aéreas que se manifesta clinicamente por episódios recorrentes de dispnéia, sibiância, constrição torácica e tosse. É caracterizada por hiper-responsividade brônquica e obstrução variável do fluxo aéreo, reversível espontaneamente ou com o tratamento!

Todos os pacientes com asma estão sob risco de apresentar uma exacerbação da doença. A crise asmática é caracterizada por aumento progressivo na dispneia, na tosse, na sibilância ou na constrição torácica, acompanhada de diminuição do fluxo expiratório, quantificada por medidas funcionais pulmonares como o pico de fluxo expiratório (PFE) ou como o volume expiratório forçado no primeiro segundo $\left(V E F_{1}\right)$. A gravidade da crise pode variar de um quadro leve até um quadro ameaçador à vida. Geralmente, a deterioração progride ao longo de horas, dias ou até semanas. Menos frequentemente, a crise progride de forma rápida, em minutos, colocando em risco a vida do paciente?

A gravidade da apresentação clínica inicial e a resposta ao tratamento com broncodilatadores beta ${ }_{2}$-agonistas inalatórios definirão qual o melhor cenário para manejo da crise asmática. Os casos com evolução precária necessitarão de manejo no departamento de emergência ou no pronto-socorro. O manejo rápido e eficiente da crise asmática pode alterar favoravelmente o desfecho desta situação clínica, de forma a se fazer necessário a sua abordagem sistematizada e diferenciada pelos serviços de emergência ${ }^{3}$.
Desta forma, o manejo da asma aguda, em especial na sala de emergência, assume importância relevante e têm sido tema de extensa revisão na literatura recente ${ }^{2-5}$. O nosso objetivo neste artigo foi revisar a literatura, de forma a estruturar as etapas envolvidas no manejo da asma aguda na sala de emergência.

\section{Epidemiologia}

A asma constitui-se em uma emergência médica muito comum, compreendendo cerca de $5 \%$ dos atendimentos médicos nos serviços de emergência².

No Brasil, ocorrem anualmente cerca de 350.000 internações por asma, constituindo-se na quarta causa de hospitalização pelo Sistema Único de Saúde (SUS), o que corresponde a 2,3\% do total de internações 6 .

Em muitos países, a mortalidade por asma aumentou a partir de 1960, com dois picos na incidência: décadas de 60 e 80. Atingiu um platô e diminuiu a partir da década de 90 . Essa tendência de declínio parece refletir um melhor manejo da doenç̧7. No Brasil, a mortalidade por asma em adultos jovens variou de 0,276 a I,034 / I00.000 ao ano. Houve um aumento no período de 1970 a 1992 em crianças e em adultos jovens, mas a tendência estabilizou com tendência a diminuição a partir da metade da década de $90^{8}$.

\section{Fisiopatogenia e fisiopatologia}

A crise de asma é causada por diferentes gatilhos que induzem inflamação nas vias aéreas e provocam broncoespasmo. Esses

1. Professor Adjunto, Departamento de Medicina Interna, Faculdade de Medicina, Universidade Federal do Rio Grande do Sul (UFRGS), Serviço de Pneumologia, Hospital de Clínicas de Porto Alegre (HCPA), Porto Alegre, RS, Brasil.

2. Médico pneumologista, aluno do Programa de Pós-graduação em Medicina-Pneumologia da UFRGS. Porto Alegre, RS, Brasil 


\begin{tabular}{lcc}
\hline & Tabela I - Diagnóstico diferencial de asma aguda no adulto & \\
\hline Vias aéreas superiores & Vias aéreas inferiores & Outras \\
\hline Edema de laringe & Aspiração & Insuficiência cardíaca esquerda \\
Corpo estranho & Corpo estranho & Tumor carcinoide \\
Neoplasia & Neoplasia & Embolia pulmonar \\
Estenose traqueal & Estenose brônquica & Pneumonias eosinofllicas \\
Paralisia de cordas vocais & Exacerbação da DPOC & Reações alérgicas ou anafiláticas \\
Disfunção de cordas vocais & Pneumonia & Exposição a fumaç̧a ou vapores tóxicos \\
& & Envenenamento por organofosforado \\
& & Refluxo gastroesofágico \\
\hline
\end{tabular}

Adpatado de Dalcin PTR et al. ${ }^{4}$

desencadeantes variam de pessoa para pessoa e de momento para momento na história da doença. Os principais desencadeantes da crise asmática, identificados na prática clínica, são: alérgenos inalatórios, infecção viral das vias aéreas, poluentes atmosféricos, exercício físico, mudanças climáticas, alimentos, aditivos, drogas e estresse emocional. Menos frequentemente, outros fatores também podem contribuir como desencadeante: rinite alérgica, sinusite bacteriana, polipose nasal, menstruação, refluxo gastroesofágico e gestaçãa².

O mecanismo pelo qual a limitação aguda do fluxo aéreo é desencadeada varia de acordo com o fator desencadeante. A broncoconstrição induzida pelos alérgenos resulta da produção de mediadores inflamatórios dependentes da liberação de imunoglobulina E pelos mastócitos. Entretanto, a broncoconstrição aguda pode também ocorrer devido à hiper-responsividade das vias aéreas a uma variedade de estímulos não-alérgicos. Nessa situação, os mecanismos envolvidos na bronconstrição aguda são, além dos mediadores inflamatórios, os reflexos neurais desencadeados por estimulação central e local. Qualquer que seja o fator desencadeante, a via final comum desse processo resulta em contração da musculatura lisa das vias aéreas, aumento na permeabilidade capilar, extravasamento capilar, edema e espessamento da mucosa brônquica².

O estreitamento variável das vias aéreas, em decorrência da inflamação brônquica e do aumento do tônus brônquico, é característica da crise asmática e responsável pelo aumento da resistência ao fluxo aéreo, hiperinflação pulmonar e desuniformidade ventilação/ perfusão. Com a progressão da obstrução ao fluxo aéreo na crise asmática grave, a insuficiência respiratória é consequência do aumento do trabalho respiratório, da troca gasosa ineficaz e da exaustão dos músculos respiratório 2,9 .

\section{0 manejo na sala de emergência}

As evidências atuais mostram que o manejo da asma aguda na sala de emergência concentra etapas fundamentais que podem determinar o desfecho desta situação clínica. Estas etapas incluem o diagnóstico, a avaliação da gravidade, o tratamento, o rastreamento de complicações e a definição do local onde se realizará a complementaçãa terapêutica ${ }^{4}$.

\section{Diagnóstico}

Em geral, o diagnóstico da crise de asma aguda na sala de emergência se constitui em um processo clínico rápido e fácil. O paciente apresenta-se com algum grau de dispneia, desconforto torácico e sibilância, às vezes acompanhados de tosse e expectoração. Os sintomas apresentam duração variável de horas a dias, porém com exacerbação nas últimas horas, o que motivou sua vinda à emergência. Frequentemente, há o relato de episódios semelhantes previamente, podendo haver identificação de fatores desencadeantes e descrição de resposta sintomática favorável a medicações broncodilatadoras ${ }^{4}$.

Entretanto, deve-se lembrar que "nem tudo que sibila é asma" e que várias condições podem mimetizar asma aguda no adulto (Tabela I). Por outro lado, deve-se ressaltar que a exacerbação asmática grave pode ocorrer sem a presença de sibilos, situação que pode não ser diagnosticada ou ter sua gravidade subestimada ${ }^{4}$.

\section{Avaliação da gravidade}

A avaliação da gravidade da crise asmática visa identificar o paciente de maior risco que requer monitorização e tratamento mais agressivo para evitar a insuficiência respiratória e morte4.

Os dados da história pregressa que identificam o paciente com uma doença com maior risco incluem: intubação e ventilação mecânica prévias, acidose respiratória sem necessidade de intubação, duas ou mais hospitalizações por asma no último ano, hospitalização no último mês, tratamento crônico com corticoide no último ano, suspensão recente da corticoterapia, tratamento médico inadequado, má aderência ao tratamento e problemas psicológicos ou psicossociais ${ }^{2-4}$. O fator isolado que se associa com maior risco para um desfecho fatal da crise é a necessidade prévia de intubação e ventilação mecânica ${ }^{10}$. Cerca de $23 \%$ dos asmáticos que sobreviveram após serem submetidos a ventilação mecânica morreram por asma nos próximos seis anos". Embora a história positiva para fatores de risco possa contribuir significativamente na identificação do mau prognóstico da crise asmática e de sua evolução para um estado de mal asmático, ela está presente em apenas 36\% dos casos de asma fatal e em $6 \%$ dos casos de estado de mal asmático. Assim, devemos salientar que a ausência desses fatores não contribui na avaliação de risco da crise? ${ }^{2}$.

Os dados da história da crise atual que denotam gravidade incluem duração prolongada dos sintomas, demora em buscar auxilio médico, exacerbação em vigência de corticoterapia e tratamento broncodilatador adequados, recidiva dos sintomas após horas ou poucos dias de atendimento de emergência e presença de comorbidades ${ }^{3,4}$.

No exame clínico, a aparência geral do paciente fornece o indicador mais rápido de gravidade: postura em pé, com incapacidade de assumir 
a posição supina. Os seguintes sinais denotam a gravidade da limitação do fluxo aéreo: frequência cardíaca maior que 120 batimentos/minuto, frequência respiratória maior que 30 movimentos/minuto, pulso paradoxal maior que $12 \mathrm{~mm} \mathrm{Hg}$, incapacidade de falar, fala monossilábica, uso da musculatura acessória da respiração, sudorese, tórax silencioso, cianose e alteração do sensório. O clássico achado da sibilância associase pouco com o grau de limitação do fluxo aéreo ${ }^{5}$.

Entretanto, o médico não deve confiar apenas nos parâmetros clínicos para a avaliação da gravidade da exacerbação da asma, pois a estimativa clínica tende a subestimar o grau de limitação do fluxo aéreo e a correlação dos parâmetros clínicos com os testes de função pulmonar não é boa ${ }^{12,13}$. Além disso, os sintomas e sinais que expressam gravidade na asma aguda desaparecem frente a pequenos aumentos no VEF, e os pacientes podem ser julgados clinicamente bem na vigência de importante limitação do fluxo aéreo ${ }^{3}$.

Desta forma, se faz necessário uma determinação objetiva da gravidade da obstrução do fluxo aéreo na asma aguda. Ela pode ser realizada pela medida do PFE ou pela espirometria. Estes testes de função pulmonar se constituem no padrão áureo de avaliação do fluxo aéreo nestas circunstâncias e são passíveis de uso em todos os pacientes com idade maior que seis anos de idade ${ }^{14}$. Na prática, a medida do PFE é mais fácil de ser realizada e o aparelho é bem mais barato. A medida da limitação do fluxo aéreo através da determinação do VEF, pela espirometria ou do PFE pelo aparelho de PFE fornecem dados objetivos para avaliar a gravidade da crise, quantificar a resposta ao tratamento e decidir sobre a internação hospitalar. As manobras para a realização destes testes são fáceis e seguras para a grande maioria de pacientes com asma aguda, embora em alguns pacientes intensamente dispneicos elas possam piorar o broncoespasmo, sendo prudente postergar o seu emprego nestas situações ${ }^{15}$. A asma grave é caracterizada por um VEF, ou PFE menor que $50 \%$ do previsto, sendo que valores menores que $30 \%$ do previsto (VEF $<1$ I ou PFE $<100 \mathrm{l} / \mathrm{min}$ ) se constituem em alerta para extrema gravidade e iminente exaustão ${ }^{3}$. Esta avaliação funcional deve ser associada à medida da saturação do oxigênio pela oximetria de pulso $\left(\mathrm{SpO}_{2}\right)$. No momento de admissão, o paciente é identificado como tendo risco de evolução para insuficiência respiratória quando apresentar PFE menor que 33\% do previsto ou a $\mathrm{SpO}_{2}$ menor que $92 \%$. Pelo contrário, quando o paciente tiver PFE maior que 33\% do previsto e $\mathrm{SpO}_{2}$ maior que $92 \%$, o risco de evoluir para insuficiência respiratória é mínimo ${ }^{16}$.

Além disso, a avaliação objetiva repetida ao longo do tratamento é muito útil na identificação dos pacientes que falham em apresentar resposta funcional pulmonar ao tratamento broncodilatador com 0 beta -agonista de curta ação inalatório. Cerca de I/3 a I/5 dos pacientes asmáticos atendidos na emergência apresentam uma resposta precária ao broncodilatador inalatório, falhando em atingir um VEF, ou PFE de $45 \%$ do previsto na primeira hora de manejo. Esses pacientes também constituem um grupo de risco para evolução para insuficiência respiratória, sendo fundamental que sejam prontamente identificados no OS 3 .

A gasometria arterial estará indicada quando a $\mathrm{SpO}_{2}$ for menor que 92\% na avaliação inicial em ar ambiente, quando houver deterioração clínica a despeito do tratamento broncodilatador pleno ou na iminência de intubação e ventilação mecânica².

\section{Tratamento}

As metas do tratamento da asma aguda grave são: a) manter adequada saturação de oxigênio arterial através da suplementação de oxigênio, b) aliviar a obstrução do fluxo aéreo através de repetidas administrações de broncodilatadores, c) reduzir a inflamação das vias aéreas e d) prevenir futuras recidivas com a administração de corticosteroides sistêmicos ${ }^{2}$.

3.I Oxigenoterapia: oxigênio deve ser administrado imediatamente para todos os pacientes com asma aguda que apresentarem $\mathrm{SpO}_{2}$ menor que $92 \%$, através de cânula nasal com fluxo de 2 a $3 \mathrm{l} / \mathrm{min}$. No paciente com asma aguda "pura", o risco de induzir depressão respiratória é insignificante. Nos casos mais graves, podem ser utilizados fluxos mais elevados de 4 a $5 \mathrm{l} / \mathrm{min}$ ou máscara de Venturi. A oxigenoterapia deverá ser monitorizada pela oximetria de pulso. Em pacientes com comorbidades como doença cardíaca, ou pacientes gestantes, a SpO deve ser mantida acima de $95 \%{ }^{6}$.

3.2 Broncodilatadores beta 2 -agonistas de curta ação: constituem a terapêutica de primeira linha para o tratamento da crise asmática sendo que a administração inalatória é a via de eleição 1,6. A administração das medicações beta -agonistas pela via inalatória pode ser feita por meio de aerossol dosimetrado (spray) ou por nebulizador a fluxo contínuo de ar comprimido. A administração por spray acoplado a espaçador produz broncodilatação tão eficiente quanto à obtida com a administração por nebulizador a fluxo contínuo. A incidência de tremor, palpitações e ansiedade é maior no grupo que utiliza os nebulizadores a fluxo contínuo, refletindo maior absorção sistêmica do broncodilatador ${ }^{17}$. Assim, o uso do spray acoplado ao espaçador tem sido recomendado como a forma inicial de administrar os broncodilatadores beta 2 -agonistas a pacientes com asma aguda, ficando os nebulizadores a fluxo contínuo como segunda opção em pacientes graves ou sem coordenação motora necessária para o uso do spray. As doses preconizadas são: salbutamol spray ( $100 \mathrm{mcg} /$ /ato) 4 - 8 jatos a cada 20 min por três doses e, após, de hora em hora até melhora clínica e funcional; ou salbutamol solução para nebulização (5 $\mathrm{mg} / \mathrm{ml}$ ) 2,5 - $5 \mathrm{mg}$ a cada 20 min por três doses e, após, de hora em hora até melhora clínica e funcional2. O uso prévio domiciliar dos beta-agonistas de curta ou longa ação não deve limitar o tratamento pleno na sala de emergência com estas medicações ${ }^{5}$.

As evidências para o uso intravenoso ou subcutâneo dos beta ${ }_{2}{ }^{-}$ agonistas ou da adrenalina na asma aguda grave são precárias e controversas. Porém, em situações especiais, esta forma de administração pode ser considerada como medida terapêutica heróica em pacientes jovens (idade menor que 40 anos) em que o tratamento inalatório não foi possível ou não resultou em resposta broncodilatadora significativa, na tentativa de postergar a intubação endotraqueal ou de reduzir a hiperinflação progressiva em pacientes submetidos a ventilação mecânica?.

3.3 Broncodilatadores anticolinérgicos: são broncodilatadores menos potentes e com início de ação mais lento do que os beta ${ }_{2}-$ agonistas de curta ação. O seu uso, combinado aos beta ${ }_{2}$-agonistas, tem sido preconizado na asma aguda grave na sala de emergência em crianças e adultos com VEF, menor que $50 \%$ do previsto. Sua utilização reduz as taxas de internação hospitalar em $30 \%$ a $60 \%{ }^{18}$. Não acarreta nenhum aumento de efeitos colaterais. A droga anticolinérgica padrão 
empregada no tratamento da asma aguda na emergência é o brometo de ipratrópio (soluçãao para inalaçãa com 0,25 mg/ml ou spray com $0,020 \mathrm{mg} /$ /ato). As doses preconizadas são: nebulização de 0,25 a 0,5 mg a cada 20 min por três doses e, após, a cada 2 - 4 horas até melhora clínica e funcional; ou inalação de 4 - 8 jatos a cada 20 min por 3 doses e, após, a cada 2 - 4 h até melhora clínica e funcional ${ }^{2,9}$.

3.4 Corticosteroides sistêmicos: constituem-se em componente essencial no tratamento da crise asmática atendida na emergência. $\mathrm{O}$ atraso no uso de corticosteroides ou a não administração é citado como fator de risco para a morte durante a exacerbação da asma. É a única medicação que evita a recidiva dos sintomas e reduz a taxa de hospitalização, sendo importante seu uso ainda na primeira hora da crise asmática ${ }^{19}$. Não há evidências que demonstrem benefício da via intravenosa sobre a oral, de forma que a via oral seria a primeira escolha. Entretanto, como os estudos não incluíram pacientes de muita gravidade, parece razoável reservar a via intravenosa para aqueles pacientes no extremo da gravidade. As doses preconizadas são: prednisona I mg/kg via oral até $6 / 6 \mathrm{~h}$, hidrocortisona 2-3 $\mathrm{mg} / \mathrm{kg}$ intravenosa até $4 / 4 \mathrm{~h} \mathrm{e}$ metilprednisolona 60-125 mg intravenosa até 6/6 h2,3,9. Tanto o intervalo da dose subsequente de corticosteroide como a sua dose total diária irão depender da resposta clínica do paciente nas primeiras horas. Esta posologia, bem como a equivalência entre os corticosteroides sistêmicos (metiprednisolona, hidrocortisona ou prednisona), não está fundamentada em ensaios clínicos e é regida atualmente por recomendações dos consensos nacionais e internacionais, os quais apresentam doses muito díspares ${ }^{1,6,16}$.

3.5 Corticosteroides inalatórios: o uso de corticoides inalatórios para o tratamento da asma aguda na emergência tem tido evidências crescentes. Uma revisão sistemática sugeriu que o uso precoce de corticosteroide inalatório reduz as admissões e melhora a função pulmonar em 8\% do PFE comparado com placebo. Entretanto, vários dos estudos dessa revisão não utilizaram corticosteroides sistêmicos, dificultando a determinação do benefício aditivo ${ }^{20}$. Mais recentemente, um ensaio clínico comparou o tratamento com fluticasona 250 mcg (spray + espaçador) dois jatos a cada 10 min (3.000 mcg / h) durante três horas de estudo versus hidrocortisona $500 \mathrm{mg} \mathrm{IV}$, associados ao tratamento padrão. Os pacientes tratados com fluticasona tiveram, ao final das três horas, um melhor desfecho funcional pulmonar ${ }^{21}$. Porém, ainda permanecem questões a serem esclarecidas quanto ao uso dos corticosteroides inalatórios na asma aguda: benefício da corticoterapia inalatória em conjunto com a corticoterapia sistêmcia, droga ideal, dose e frequência de administração, avaliação de desfechos mais longos que três horas ${ }^{1,6}$.

3.6 Sulfato de magnésio: o uso do sulfato de magnésio como medida terapêutica adjuvante para o tratamento da asma aguda grave tem recebido atenção crescente na literatura. $O$ sulfato de magnésio diminui as taxas de internação e melhora a função pulmonar em pacientes com asma grave com $\mathrm{VEF}_{1}<25 \%$ do previsto. Também parece diminuir a necessidade de intubação e ventilação mecânica. Entretanto, seu uso permanece controverso. No conhecimento atual, o sulfato de magnésio tem sua utilização justificada na asma aguda refratária às medidas terapêuticas convencionais, na tentativa de evitar a insuficiência respiratória e a consequente intubação e ventilação mecânica. A dose preconizada para esta situação clínica é de $2 \mathrm{~g}$ ( $4 \mathrm{ml}$ de sulfato de magnésio a 50\%), diluída em $50 \mathrm{ml}$ de soro fisiológico e administrada em 20 minutos, podendo ser repetido $2 \mathrm{~g}$ em 20 minutos 22,23 .

3.7 Xantinas: A aminofilina que já foi considerada a arma terapêutica fundamental no manejo da asma aguda perdeu, nos últimos anos, esta posição de destaque. É um erro frequente considerar, atualmente, a aminofilina como a principal medicação no tratamento da asma aguda. O consenso atual da literatura é de que esta medicação não tem mais papel no manejo de rotina desta situação clínica, ${ }^{1,6,16}$. Foi demonstrado que o uso da aminofilina na asma aguda não oferece efeito broncodilatador adicional ao tratamento com beta-agonista, mas aumenta significativamente os efeitos colaterais como náusea, vômito, tremor e ansiedade ${ }^{24,25}$. Entretanto, esta medicação ainda encontra indicação restrita no manejo de pacientes em estado de mal asmático ou doença refratária, isto é, naqueles pacientes em que a condição respiratória continua a se deteriorar, mesmo após 30 a 60 minutos de tratamento pleno com broncodilatadores beta ${ }_{2}$-adrenérgicos e corticosteroides ${ }^{26}$. A dose preconizada nesta situação clínica é de um bolo inicial de 5 a $6 \mathrm{mg} / \mathrm{kg}$, administrado em 20 minutos, seguido de uma infusão contínua de 0,6 a $0,9 \mathrm{mg} / \mathrm{kg} / \mathrm{hora}$. Nos pacientes já em uso de teofilina, não se administra a dose de ataque e se inicia a infusão contínua. Lembrar que, idealmente, o nível sérico deve ser monitorizado em 6 a 12 horas, com meta de mantê-lo entre 8 a 15 mcg/ $\mathrm{m}^{27}$. A dose de manutenção deve ser reduzida a $0,4 \mathrm{mg} / \mathrm{kg} /$ hora nos pacientes idosos e a $0,2 \mathrm{mg} / \mathrm{kg} /$ hora nos pacientes com insuficiência cardíaca congestiva ou doença hepática. Nos pacientes obesos, existe um maior volume de distribuição e uma maior meia-vida, de forma que a dose de ataque é calculada pelo peso total e a de manutenção pelo peso ideal 15 .

3.8 Heliox: é uma mistura de hélio e oxigênio que apresenta uma densidade gasosa menor que a do ar ambiente. As proporções desta mistura hélio: $\mathrm{O}_{2}$ são geralmente de 80:20, 70:30 ou 60:40. Pode ser utilizado na asma aguda pelo sistema de máscara facial, ou em paciente intubado, conectado ao ramo inspiratório do circuito ventilatório 5 . Teoricamente, a menor densidade desta mistura gasosa permite uma menor resistência a seu fluxo nas vias aéreas se comparado ao oxigênio/ ar sem heliox, reduzindo o trabalho respiratório, retardando a fadiga dos músculos respiratórios e ganhando tempo para a ação da terapêutica broncodilatadora e anti-inflamatória. Tem sido estudada como uma alternativa para postergar a exaustão respiratória, enquanto se administra o tratamento broncodilatador pleno na asma aguda grave ${ }^{28}$. Entretanto, uma revisão sistemática recente não mostrou benefício de sua utilização na asma aguda na emergência 29 .

3.9 Ventilação mecânica não-invasiva: a experiência com a ventilação não-invasiva (VNI) com pressão positiva nas vias aéreas em dois níveis (bilevel), isto é, o suporte pressórico inspiratório (IPAP) com pressão expiratória final positiva (EPAP), e a sua utilidade bem definida na doença pulmonar obstrutiva crônica e em casos iniciais de síndrome da angústia respiratória do adulto, somada à necessidade de postergar a intubação na asma aguda grave enquanto se aguarda a resposta ao corticoide, despertou interesse em utilizar esta modalidade terapêutica na asma brônquica ${ }^{30,31}$. Os mecanismos aventados para esta melhora 
são: redução do trabalho respiratório (manutenção dos sacos alveolares patentes no final da expiração e auxílio ao esforço inspiratório), recrutamento dos músculos expiratórios para auxiliar na inspiração, redução da hiperinsuflação pulmonar com inspiração mais eficiente e amenização das consequências hemodinâmicas adversas da grande negatividade da pressão pleural inspiratória ${ }^{30}$. Alguns estudos abertos e não-randomizados sugeriram benefícios semelhantes aos encontrados na síndrome da angústia respiratória do adulto e na doença pulmonar obstrutiva crônica ${ }^{30}$. Sorosky et al. ${ }^{32}$ realizaram o único estudo randomizado e controlado até o momento. Neste estudo foram randomizados 30 pacientes com agudização da asma que foram alocados em dois grupos, um usando VNI tipo bilevel e outro usando "VNI-placebo" (grupo controle, consistia na utilização de VNI com pressões ins e expiratórias de $\left.\mathrm{I} \mathrm{CmH}_{2} \mathrm{O}\right)$. Foi demonstrado que os pacientes que receberam VNI por três horas consecutivas apresentaram melhora mais rápida dos sintomas, aumento do VEF, e diminuição da necessidade de internação em relação ao grupo controle. Revisão sistemática recente da Cochrane sobre o tema não encontrou nenhum outro estudo relevante, concluindo que o uso da VNI para o tratamento da exacerbação grave da asma parece promissor, porém o único estudo publicado e o limitado número de pacientes avaliados não permitem a recomendação definitiva para seu uso ${ }^{33}$. Atualmente aceita-se o uso da $\mathrm{VNI}$, em conjunto com o tratamento medicamentoso convencional, para o manejo de pacientes selecionados e com exacerbação aguda e grave da asma ${ }^{34}$.

3. I0 Intubação endotraqueal e ventilação mecânica: as indicações absolutas para intubação endotraqueal frente a uma crise asmática são: parada cardíaca, parada respiratória ou significante alteração no estado mental. Entretanto a decisão de proceder à intubação na crise asmática nem sempre é tão fácil como nas indicações absolutas. Mais frequentemente, a intubação endotraqueal é necessária em pacientes que apresentam exaustão progressiva. Nestas circunstâncias, a decisão de intubar pode ser muito difícil e o médico terá que colocar em julgamento o risco da intubação e da ventilação mecânica versus o risco de deterioração do paciente até o ponto de parada cardiorrespiratória. $\mathrm{Na}$ ausência das indicações absolutas, a intubação endotraqueal só deverá ser realizada após uma tentativa de tratamento broncodilatador em doses plenas. A acidose respiratória, isoladamente, sem levar em conta o curso evolutivo da crise, não é indicador de intubação endotraqueal e ventilação mecânica ${ }^{4,5}$. Assim, mesmo quando a $\mathrm{PaCO}_{2}$ inicial é maior que 55 a $60 \mathrm{~mm} \mathrm{Hg}$, muitos pacientes podem ser manejados sem intubação e ventilação mecânica ${ }^{14}$.

3.II Outras terapias: um conjunto novo de medidas terapêuticas tem sido apontado como tendo possível benefício na asma aguda na emergência. Dentre elas estão o montelucaste intravenoso, o zafirlucaste via oral, o levalbuterol nebulizado e o sulfato de magnésio isotônico nebulizado. Entretanto, as evidências de possível benefício ainda são limitadas para que essas opções terapêuticas tenham seu papel definido dentro do tratamento padrão da asma aguda grave ${ }^{1-3}$.

\section{Rastreamento de complicações}

Enquanto se trata a broncoconstrição aguda, a atenção médica deve também dirigir-se ao rastreamento das possíveis complicações que

\begin{tabular}{ll}
\hline & Tabela 2 - Complicações na asma aguda grave \\
\hline Complicação & Método diagnóstico \\
\hline Pneumotórax & Rx de tórax \\
Pneumomediastino & Rx de tórax \\
Enfisema subcutâneo & Rx de tórax \\
Pneumopericárdio & Rx de tórax \\
Atelectasia & Rx de tórax \\
Pneumonia & Rx de tórax, hemograma, gram e cultural do escarro \\
Toxicidade da teofilina & Dosagem sérica da teofilina \\
Hipocalemia & Dosagem sérica de potássio \\
Hiperglicemia & Dosagem sérica de glicose \\
Arritmia & ECG, monitorização cardíaca \\
Acidose lática & GA e lactato sérico \\
\hline
\end{tabular}

Adaptado de Dalcin PTR et al. ${ }^{4}$

ECG = eletrocardiograma; $G A=$ gasometria arterial

comumente acompanham esta situação clínica, por exemplo: pneumotórax, pneumomediastino, pneumopericárdio, atelectasia, pneumonia, intoxicação por xantina, hipopotassemia, hiperglicemia, acidose lática e arritmia. O passo inicial nesta avaliação consiste na história e exame clínico detalhado, seguido de exames complementares específicos ${ }^{4,5}$ (Tabela 2).

\section{Definição do local onde se realizará a complementação terapeuttica}

Durante o tratamento inicial da crise asmática na emergência, o médico deverá decidir o local onde se realizará a complementação terapêutical,6. Para tanto, deverá identificar quais os pacientes passíveis de prosseguir tratamento domiciliar, quais os que necessitarão internação em enfermaria e quais os que exigirão cuidados em unidade de tratamento intensivo.

Esta decisão sempre se constituiu em especial desafio e, inicialmente, era baseada apenas na avaliação da sensação subjetiva do paciente de alívio sintomático e na estimativa médica de melhora do broncoespasmo frente a um curso de tratamento broncodilatador. A partir da década de 70, vários estudos demonstraram que as provas de função pulmonar contribuíam na identificação dos pacientes de risco para admissão hospitalar ou para recidiva da crise após liberação da emergência ${ }^{12,35-37}$.

Idealmente, os pacientes passíveis de liberação ao domicilio são aqueles que tiveram uma boa resposta ao tratamento broncodilatador, não apresentam sintomas ou sinais de gravidade e atingiram um PFE ou $V E F, \geq 70 \%$ do previsto 1.6 . Em situações individualizadas, o paciente que persiste com sintomatologia leve a moderada, sem sinais clínicos de gravidade ou complicação, e com PFE ou VEF, entre 50\% a 70\% do previsto também poderá receber alta da emergência?. Os pacientes com crise asmática que permanecerem com sintomatologia grave e que não atingirem valores de PFE e VEF $\geq 50 \%$ do previsto após tratamento broncodilatador pleno na emergência ou que apresentarem alguma das complicações citadas, serão candidatos à internação hospitalar 2,69. Estudo recente demonstrou que o PFE maior ou igual a 40\% do previsto após 15 minutos do ińcio do tratamento da crise de asma prediz um desfecho favoráve $\left.\right|^{37}$. A Figura I apresenta um algoritmo que resume o tratamento da asma aguda na emergência e os critérios de alta e de internação. 


\section{Figura I - Algoritmo para manejo da asma aguda em adultos na sala de emergência.}

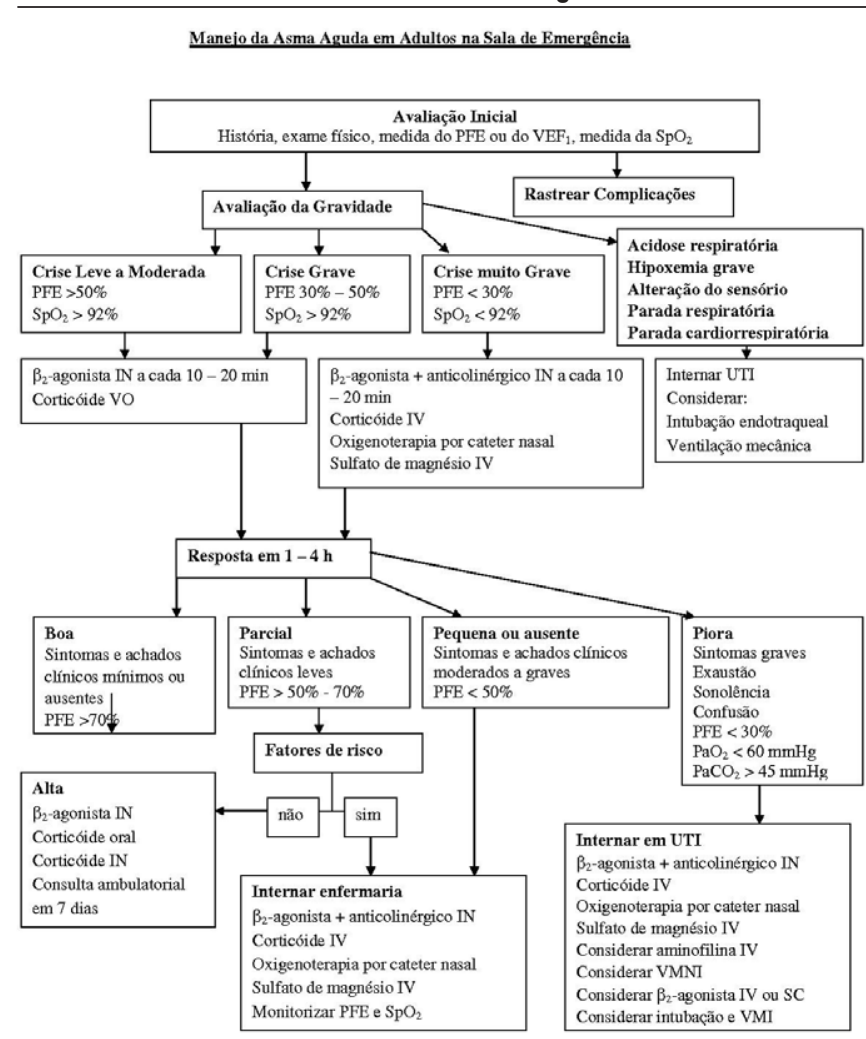

Adaptado de Dalcin PTR et al.,439

$\mathrm{PFE}=$ pico de fluxo expiratório; $\mathrm{VEF} \mid$ = volume expiratório forçado no primeiro segundo; $\mathrm{SpO} 2$ = saturação de oxigênio pela oximetria de pulso; IV = intravenoso; SC = subcutâneo; VO = via oral; $\mathrm{PaO} 2$ = pressão parcial de oxigênio no sangue arterial; $\mathrm{PaCO}$ = pressão parcial de gás carbônico no sangue arterial; UTI = unidade de tratamento intensivo; VMNI = ventilação mecânica não-invasiva; VMI= ventilação mecânica invasiva.

Os seguintes critérios indicam necessidade de internação em UTI: deterioração progressiva, a despeito de tratamento pleno; hipoxemia (pressão parcial de oxigênio no sangue arterial < 60 mm Hg ou SpO, $<90 \%$ com fração de oxigênio inalado > 0,5); hipercapnia (pressão parcial de gás carbônico no sangue arterial > 45 mm Hg); confusão mental ou sonolência; inconsciência; parada respiratória ou cardiorrespiratória; ventilação mecânica ${ }^{3,6}$.

\section{Orientação por ocasião da liberação do pronto-socorro}

É importante que, ao liberar o paciente da sala de emergência, se forneça um sumário de orientações envolvendo prescrição das medicações a serem utilizados, propósito de cada medicação, técnica correta de uso de cada dispositivo inalatório, consulta ambulatorial nos próximos sete dias e plano de ação escrito ${ }^{6}$.

O uso de corticosteroides sistêmicos após a alta da emergência é fortemente recomendado, pois está associado a menor chance de recidiva da crise (razão de chances de 0,35, favorecendo o uso de corticosteroide sistêmico, comparado com o uso de placebo) $)^{19}$. Assim, o paciente deve receber, por ocasião da alta da emergência, a prescrição de prednisona 40 - 60 mg ao dia, por sete a dez dias'.

As evidências atuais também fundamentam o uso de corticosteroide inalatório associado ao corticosteroide sistêmico, por ocasião da liberação da emergência. Uma revisão sistemática mostrou que essa combinação teve menos recidiva pós-alta que o uso de corticosteroides sistêmico isoladamente (razão de chances de 0,68, favorecendo o uso combinado do corticosteroide sistêmico com o corticosteroide inalatório ${ }^{20}$.

Não há evidências suficientes que mostrem que o corticosteroide sistêmico possa ser substituído pelo corticosteroide inalatório por ocasião da alta da emergência, de forma que a utilização da prednisona oral é imperativa nessa situação clínica 6 .

O encaminhamento ambulatorial a um especialista em asma pode reduzir o risco de recidiva e melhorar os desfechos após uma crise de asma atendida na emergência ${ }^{38}$.

\section{Prognóstico}

A grande maioria dos pacientes com crise asmática atendida na sala de emergência sobrevive ao evento. Em uma revisão de 18 estudos, foram observadas 67 mortes em 15.300 atendimentos na emergência ${ }^{3}$. Em um estudo realizado no Hospital de Clínicas de Porto Alegre, foram observadas duas mortes em 500 atendimentos ${ }^{39}$. $\mathrm{O}$ risco de morte aumenta com a intubação endotraqueal e ventilação mecânica ${ }^{3}$. Nos pacientes admitidos na UTI por crise asmática, a mortalidade média é 8,3\% $\%^{40}$.

\section{Prevenção}

A disponibilidade de cuidados ambulatoriais e a adesão ao tratamento com corticosteroide inalatório e beta 2 -agonista de longa ação reduzem as exacerbações asmáticas e as visitas à emergência ${ }^{41,42}$.

\section{Conflito de interesse: não há}

\section{SUMMARY}

Management of acute asthma in adults in the emergency ROOM: CURRENT EVIDENCE

Asthma is a disease with high prevalence in our country and worldwide. Although new therapeutic approaches have been developed recently, there seems to be a global increase in morbidity and mortality from asthma. In many institutions, asthma exacerbation is still a common medical emergency. Clinical evidence demonstrates that management of acute asthma in the emergency room entails crucial decisions that could determine the clinical outcome. In this review, the authors focus on assessment and treatment of patients with acute asthma and outline an appropriate management strategy. Diagnosis, severity assessment, treatment, complications, decision about where additional treatment will take place and orientations on discharge from the emergency will be considered. It is expected that these recommendations will help physicians to make the appropriate decisions about care of acute asthma in the emergency room. [Rev Assoc Med Bras 2009; 55(I): 82-8]

KeY Words: Asthma. Emergency medicine. Emergency treatment. Clinical procedures. 


\section{REFERÊNCIAS}

I. Global Initiative for Asthma. Global strategy for asthma management and prevention. Rev. 2006 ed. Bethesda.: National Institutes of Health, National Heart, Lung, and Blood Institute; 2006.

2. Rodrigo G], Rodrigo C, Hall JB. Acute asthma in adults: a review. Chest. 2004; | 25: | $08 \mid-102$.

3. McFadden ER Jr. Acute severe asthma. Am J Respir Crit Care Med. 2003; 1 68:740-59.

4. Dalcin PTR, Medeiros AC, Siqueira MK, Mallmann F, Lacerda M, Gazzana $M B$, et al. Asma aguda em adultos na sala de emergência: o manejo clínico na primeira hora. J Pneumol. 2000; 26:297-306.

5. Corbridge TC, Hall JB. The assessment and management of adults with status asthmaticus. Am J Respir Crit Care Med. 1995; I 51:|296-3 I6.

6. Sociedade Brasileira de Pneumologia e Tisiologia (SBPT). IV Diretrizes Brasileiras para o Manejo da Asma. J Bras Pneumol. 2006;32:447-74.

7. Comino EJ. What explains falling asthma mortality? Med J Aust. 2004; | 80:595.

8. Chatkin G, Chatkin JM, Fritscher CC, Cavalet-Blanco D, Bittencourt HR, Sears MR. Asthma mortality in southern Brazil: is there a changing trend? J Asthma. 2007:44: I33-6.

9. FitzGerald JM, Grunfeld A. Acute life-threatening asthma. In: Fitzgerald JM, Ernst P, Boulet L, OByrne PM, editors. Evidence-based asthma management. London: B.C. Decker Inc.; 2001. p.233-44.

I0. Turner MO, Noertjojo K, Vedal S, Bai T, Crump S, Fitzgerald JM. Risk factors for near-fatal asthma. A case-control study in hospitalized patients with asthma. Am J Respir Crit Care Med. 1998; I 57: I 804-9.

I I. Marquette CH, Saulnier F, Leroy O, Wallaert B, Chopin C, Demarcq JM, et al. Long-term prognosis of near-fatal asthma. Am Rev Respir Dis. |992;| 466:76-8|

12. Emerman CL, Cydulka RK. Effect of pulmonary function testing on the management of acute asthma. Arch Intern Med. 1995; 155:2225-8

13. McFadden ER, Kiser R, DeGroot WJ. Acute bronchial asthma: relations between clinical and physiologic manifestations. N Engl J Med. 1973;288:221-5

14. Nowak RM, Tokarski GF. Adult acute asthma. In: Rosen P, Barkin R, editors. Emergency Medicine: Concepts and Clinical Practice. $4^{\text {th }}$ ed. St. Luis: Mosby; 1998. p. I470-94.

15. Jagoda A, Shepherd SM, Spevitz A, Joseph MM. Refractory asthma, part 2: airways interventions and management. Ann Emerg Med. 1997; 29:275-81

16. British Thoracic Society and Scottish Intercollegiate Guidelines Network. British Guideline on the Management of Asthma. Thorax. 2003;58: I -94.

17. Cates CJ, Crilly JA, Rowe BH. Holding chambers (spacers) versus nebulisers for beta-agonist treatment of acute asthma. Cochrane Database Syst Rev. 2006;CD000052.

18. Rodrigo G], Castro-Rodriguez JA. Anticholinergics in the treatment of children and adults with acute asthma: a systematic review with metaanalysis. Thorax. 2005;60:740-6.

19. Rowe BH, Edmonds ML, Spooner CH, Diner B, Camargo CA Jr. Corticosteroid therapy for acute asthma. Respir Med. 2004;98:275-84.

20. Edmonds ML, Camargo CA, Jr., Pollack CV Jr., Rowe BH. The effectiveness of inhaled corticosteroids in the emergency department treatment of acute asthma: a meta-analysis. Ann Emerg Med. 2002;40: 1 45-54

21. Rodrigo GJ. Comparison of inhaled fluticasone with intravenous hydrocortisone in the treatment of adult acute asthma. Am J Respir Crit Care Med. 2005; 171:123|-6.

22. Rowe BH, Bretzlaff JA, Bourdon C, Bota GW, Camargo CA Jr. Intravenous magnesium sulfate treatment for acute asthma in the emergency department: a systematic review of the literature. Ann Emerg Med. 2000:36: | 8|-90.

23. Silverman RA, Osborn H, Runge J, Gallagher EJ, Chiang W, Feldman J, et al. IV magnesium sulfate in the treatment of acute severe asthma: a multicenter randomized controlled trial. Chest. 2002; 122:489-97.
24. Siegel D, Sheppard D, Gelb A, Weinberg PF. Aminophylline increases the toxicity but not the efficacy of an inhaled beta-adrenergic agonist in the treatment of acute exacerbations of asthma. Am Rev Respir Dis. 1985; 132:283-6.

25. Rodrigo C, Rodrigo G. Treatment of acute asthma. Lack of therapeutic benefit and increase of the toxicity from aminophylline given in addition to high doses of salbutamol delivered by metered-dose inhaler with a spacer. Chest. 1994; 106:107|-6.

26. Parameswaran K, Belda J, Rowe BH. Addition of intravenous aminophylline to beta2-agonists in adults with acute asthma. Cochrane Database Syst Rev. 2000;CD002742.

27. Nowak RM, Tokarski GF. Adult acute asthma. In: Rosen P, Barkin R, editors. Emergency medicine: concepts and clinical practice. $4^{\text {th }}$ ed. St. Luis: Mosby, 1998. p. 1470-94.

28. Manthous CA, Hall JB, Melmed A, Caputo MA, Walter J, Klocksieben JM, et al. Heliox improves pulsus paradoxus and peak expiratory flow in nonintubated patients with severe asthma. Am J Respir Crit Care Med. 1995; | 51:310-4.

29. Rodrigo G], Rodrigo C, Pollack CV, Rowe B. Use of helium-oxygen mixtures in the treatment of acute asthma: a systematic review. Chest. 2003; | 23:89|-6.

30. Meduri GU. Noninvasive positive-pressure ventilation in patients with acute respiratory failure. Clin Chest Med. 1996; 17:513-53.

31. Poponick JM, Renston JP, Bennett RP, Emerman CL. Use of a ventilatory support system (BiPAP) for acute respiratory failure in the emergency department. Chest. 1999; | 16:166-7|.

32. Sorosky A, Stav D, Shpirer L. A pilot prospective, randomized, placebocontrolled trial of bilevel positive airway pressure in acute asthmatic attack. Chest. 2003, 123: 1018-25.

33. Ram FS, Wellington S, Rowe B, Wedzicha JA. Non-invasive positive pressure ventilation for treatment of respiratory failure due to severe acute exacerbations of asthma. Cochrane Database Syst Rev 2005:CD004360.

34. Sociedade Brasileira de Pneumologia e Tisiologia. III Consenso Brasileiro de Ventilacao Mecanica. J Bras Pneumol. 2007;33(Supl):S5I-SI 50.

35. Banner AS, Ranchhodiel SS, Addington WW. Rapid prediction of need for hospitalization in acute asthma. JAMA. 1976;235:1337-8.

36. Fischl MA, Pitchenik A, Gardner LB. An index predicting relapse and need for hospitalization in patients with acute bronchial asthma. N Engl J Med. 1981;305:783-9.

37. Piovesan DM, Menegotto DM, Kang S, Franciscatto E, Menna Barreto SS, Dalcin PTR. Avaliação prognóstica precoce da asma aguda na sala de emergência. J Bras Pneumol. 2006;32: I-9.

38. Zeiger RS, Heller S, Mellon MH, Wald J, Falkoff R, Schatz M. Facilitated referral to asthma specialist reduces relapses in asthma emergency room visits. I Allergy Clin Immunol. 1991;87: | |60-8.

39. Dalcin PTR, Rocha PM, Franciscatto E, Kang SH, Menegotto DM, Polanczyk CA, et al. Effect of clinical pathways on the management of acute asthma in the emergency department: five years of evaluation. J Asthma. 2007:44:273-9.

40. Afessa B, Morales I, Cury JD. Clinical course and outcome of patients admitted to an ICU for status asthmaticus. Chest. 200 I; I20:1616-2I.

4l. Fernandes AK, Mallmann F, Steinhorst AM, Nogueira FL, Avila EM, Dalcin PTR, et al. Characteristics of acute asthma patients attended frequently compared with those attended only occasionally in an emergency department. J Asthma. 2003;40:683-90.

42. Dalcin PTR, Piovesan DM, Kang S, Fernandes AK, Franciscatto E, Menna Barreto SS. Factors associated with emergency department visits due to acute asthma. Braz J Med Biol Res. 2004;37: 133 I-8.

Artigo recebido: 30/1 1/07

Aceito para publicação: 17/04/08 